\title{
KULEUVEN
}

\section{Parametric state-time reduction for the transient analysis of multi-physical systems}

\section{INTRODUCTION}

This research focusses on computational cost reduction of transient simulations in many-query applications with varying model parameters, initial conditions and parametrised excitations. Figure 1 shows an overview of the presented state-time reduction approach in relation to the typical model reduction approach. The typical model reduction approach is limited to the reduction of the dimension of the states or spatial variables, whereas the state-time model order reduction (STMOR) approach reduces both the states and the temporal dimension concurrently. A major novelty in the STMOR approach is that the reduction occurs after time discretisation on an algebraic system of equations (AE).

The STMOR is in particular advantageous if the temporal dimension is significantly larger compared to the state or spatial dimension, e.g. a parameter study on a system-level, lumped parameter model at an initial design stage.

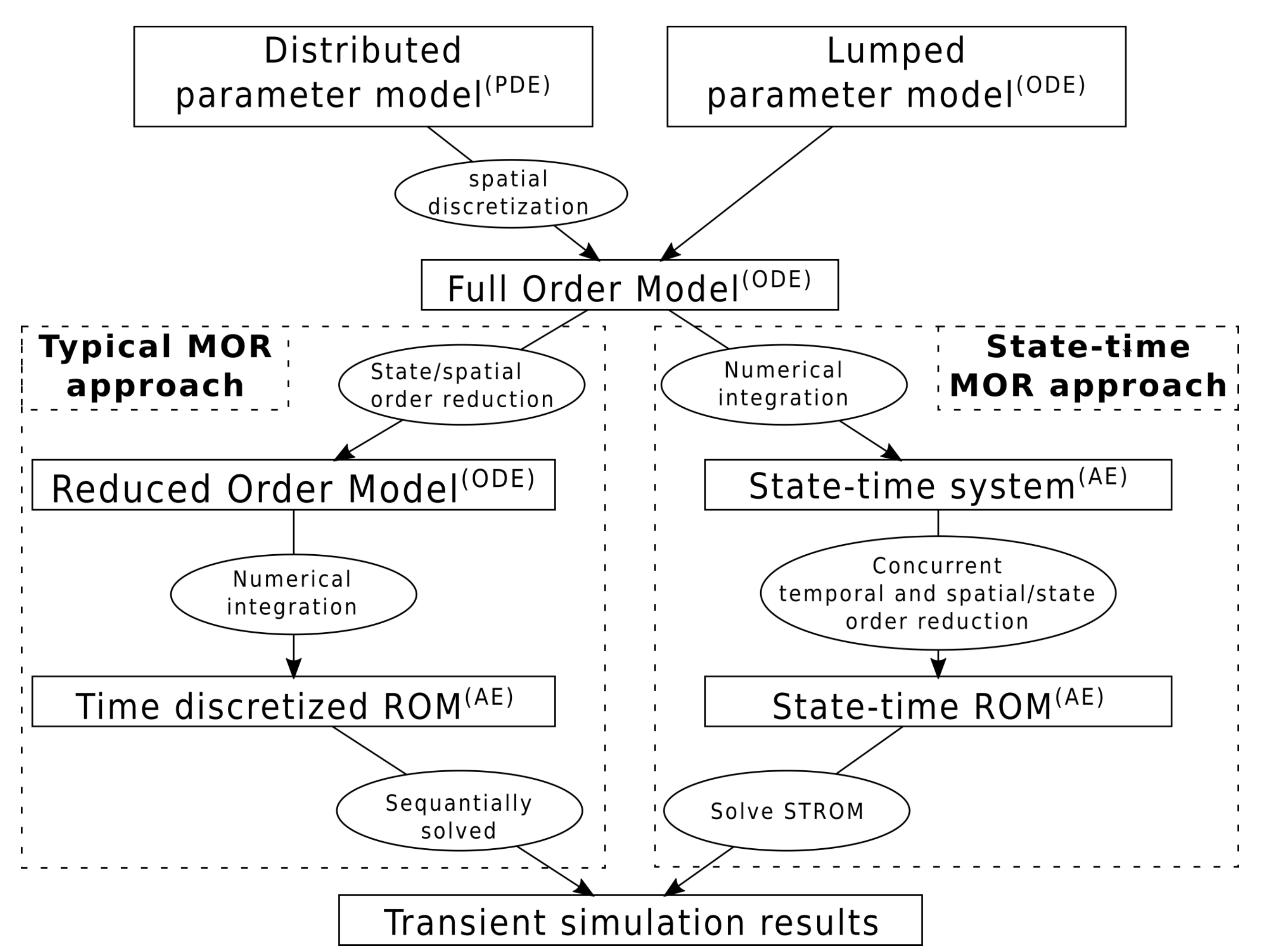

Figure 1 - Schematic overview of state-time reduction approach in comparison with the typical reduction approach described in the literature.

\section{STATE-TIME MODEL REDUCTION}

\section{State-time formulation}

Consider a parametrised, time-continuous system of first-order differential equations

$$
\dot{x}=f(x, u, p),
$$

with state $\boldsymbol{x} \in \mathbb{R}^{n_{s}}$, input $\boldsymbol{u} \in \mathbb{R}^{n_{u}}$, parameter set $\boldsymbol{p} \in \mathbb{R}^{n_{p}}$ and time $t \in \mathbb{R}^{+}$. A transient simulation approximates the time integral of (1) at discrete time instances. The complete transient simulation is assembled in a nonlinear algebraic equation with the structure

$$
D \widehat{x}=s\left(\widehat{x}, x_{0}, u, p\right) \text {, }
$$

which is called the state-time assembled system. The state-time vector $\widehat{\boldsymbol{x}}=\left\{\boldsymbol{x}^{T}[1], \ldots, \boldsymbol{x}^{T}\left[n_{t}\right]\right\}^{T} \in \mathbb{R}^{n_{s} \cdot n_{t}}$ is a concatenation of all states at each considered time instance $k$.
D

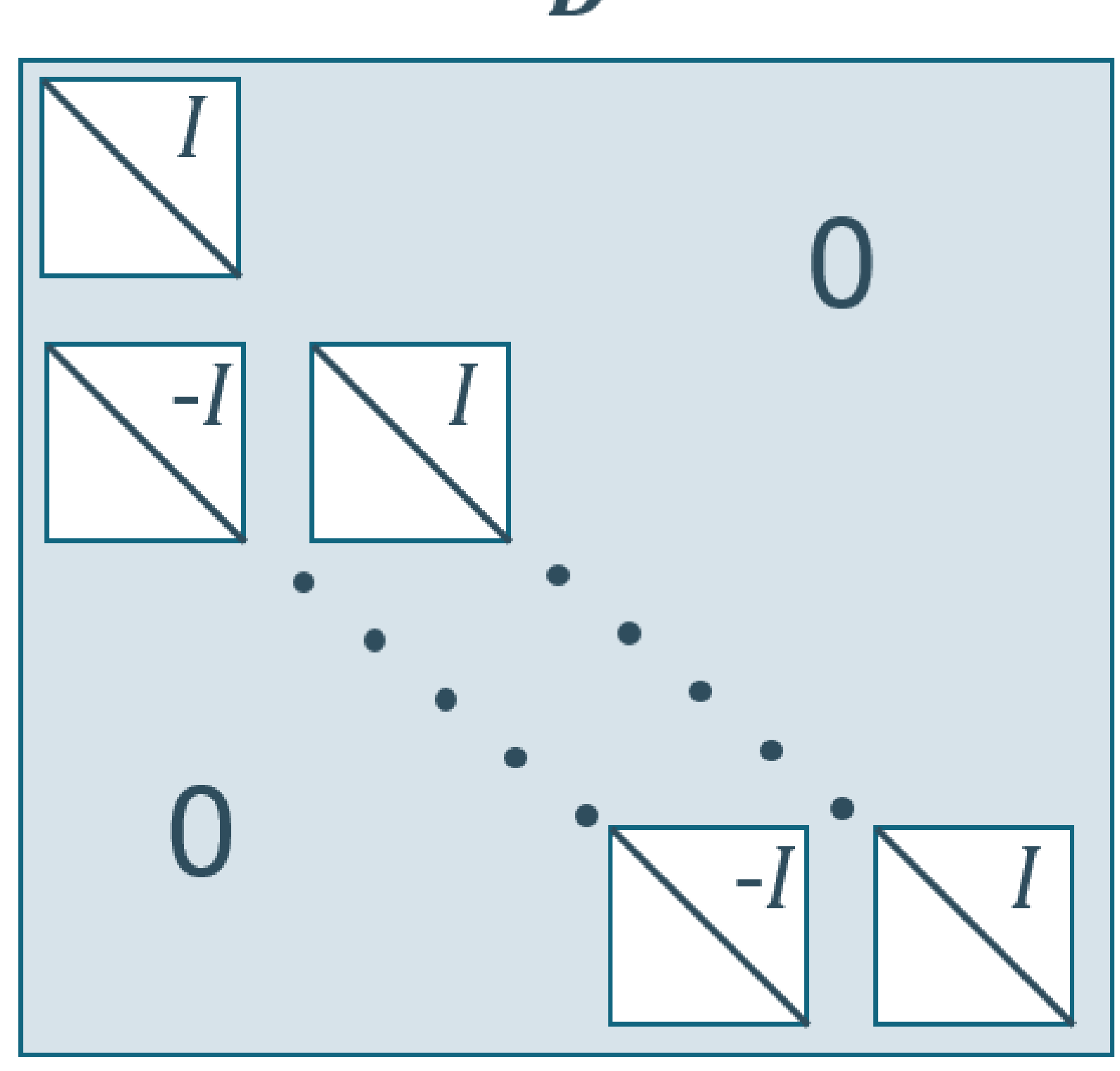

$\mathbb{R}^{n_{s} n_{t} \times n_{s} n_{t}}$

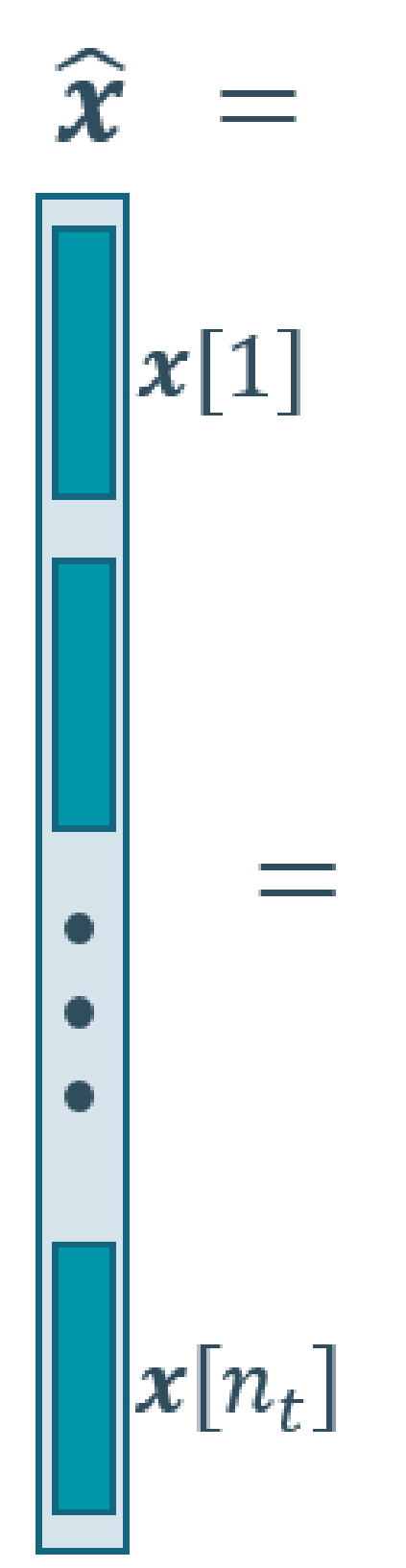

$\mathbb{R}^{n_{s} n_{t}}$

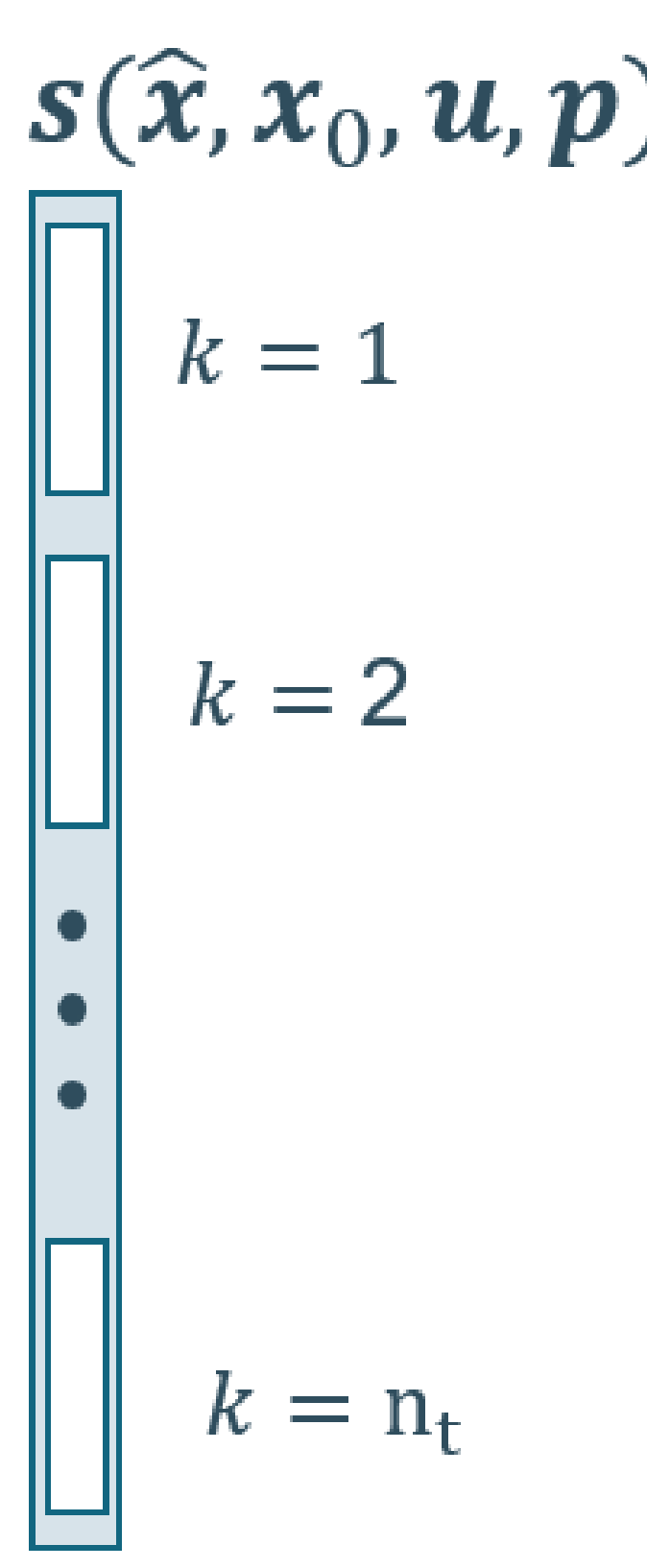

$\mathbb{R}^{n_{s} n_{t}}$
Figure 2 - A graphic representation of the state-time assembled system for an explicit solver with a time stencil of two elements.

\section{Basis calculation}

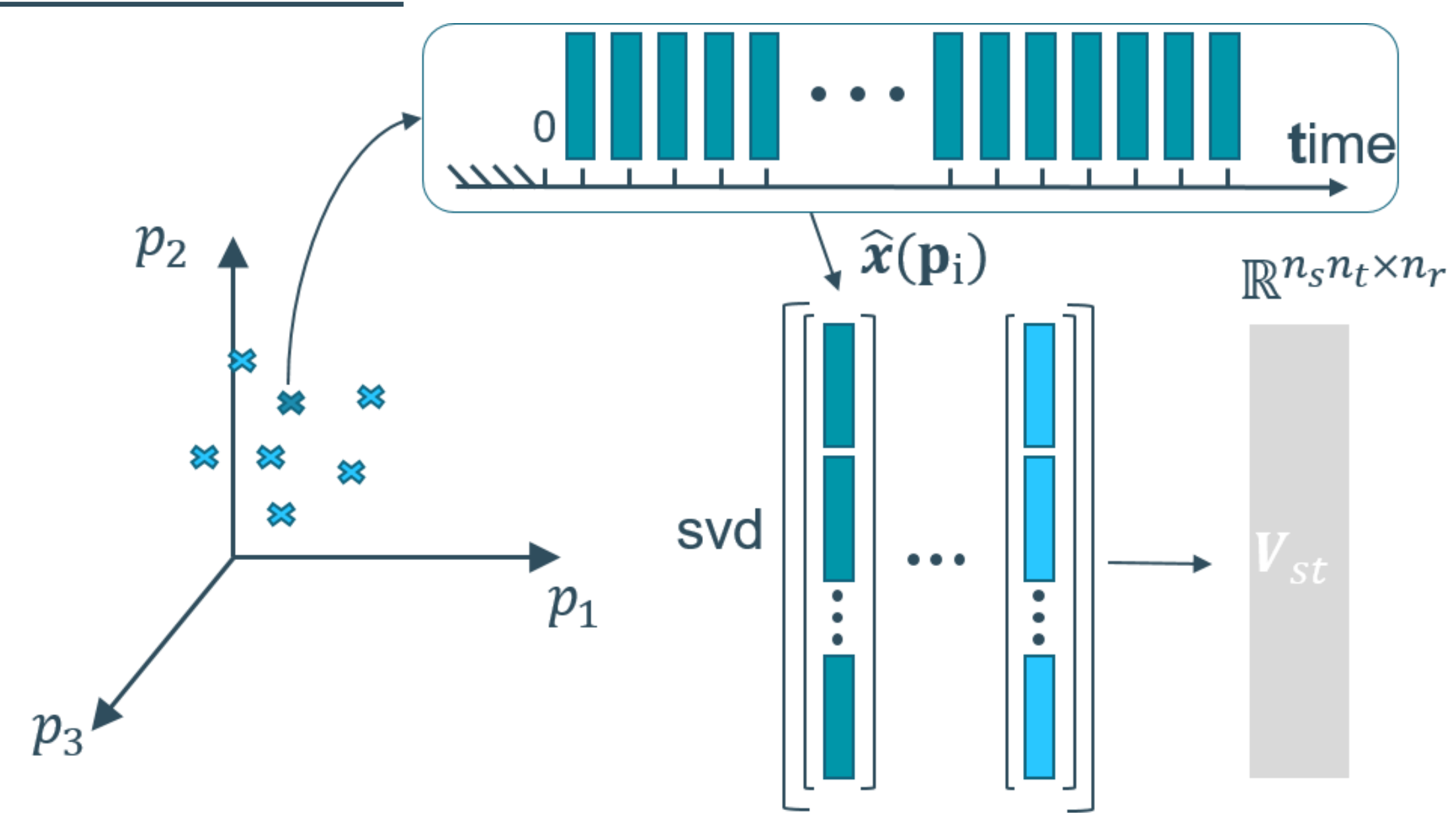

Figure 3 - Basis calculation via Proper Orthogonal Decomposition (POD) to span the solution manifold of the state-time assembled system.

\section{Reduction}

The algebraic equation (2) is reduced with a Galerkin projection yielding

$$
\boldsymbol{V}_{s t}^{T} \boldsymbol{D} \boldsymbol{V}_{s t} \boldsymbol{q}_{s t}=\boldsymbol{V}_{s t}^{T} \boldsymbol{s}\left(\boldsymbol{V}_{s t} \boldsymbol{q}_{s t}, \boldsymbol{x}_{0}, \boldsymbol{p}\right)+\boldsymbol{V}_{s t}^{T} \boldsymbol{\epsilon}
$$

To avoid an excessive computational cost to evaluate the nonlinear term $\boldsymbol{V}_{s t}^{T} \boldsymbol{s}\left(\boldsymbol{V}_{s t} \boldsymbol{q}_{s t}, \boldsymbol{x}_{0}, \boldsymbol{p}\right)$, it is a necessity to apply hyper-reduction. The Discrete Empirical Interpolation Method (DEIM) is applied, yielding

$$
\boldsymbol{V}_{s t}^{T} \boldsymbol{s}\left(\boldsymbol{V}_{s t} \boldsymbol{q}_{s t}, \boldsymbol{x}_{0}, \boldsymbol{p}\right) \approx \boldsymbol{V}_{s t}^{T} \boldsymbol{U}\left(\boldsymbol{P}^{\boldsymbol{T}} \boldsymbol{U}\right)^{-\mathbf{1}} \boldsymbol{P}^{\boldsymbol{T}} \boldsymbol{s}\left(\boldsymbol{V}_{s t} \boldsymbol{q}_{s t}, \boldsymbol{x}_{0}, \boldsymbol{p}\right) .
$$

The basis $\boldsymbol{U} \in \mathbb{R}^{n_{s} n_{t} \times n_{d}}$ is computed by pre-multiplying the decomposed matrix in figure 3 with $D$.

\section{म}

Figure 4 - Visualisation of the concurrent state and temporal dimension reduction

\section{Solve STROM}

The nonlinear state-time ROM (3), including the DEIM hyper-reduction described in (4), is solved by minimising its residue

$$
\boldsymbol{q}_{s t}=\underset{\boldsymbol{q}_{s t} \in \mathbb{R}^{n_{r}}}{\operatorname{argmin}}\left\|\boldsymbol{r}\left(\boldsymbol{q}_{s t}, \boldsymbol{p}_{i}\right)\right\|_{2}^{2}
$$

\section{NUMERICAL CASE}

The STMOR approach is demonstrated on a nonlinear drivetrain mode (figure 5), consisting of an induction motor and cardan shaft model.

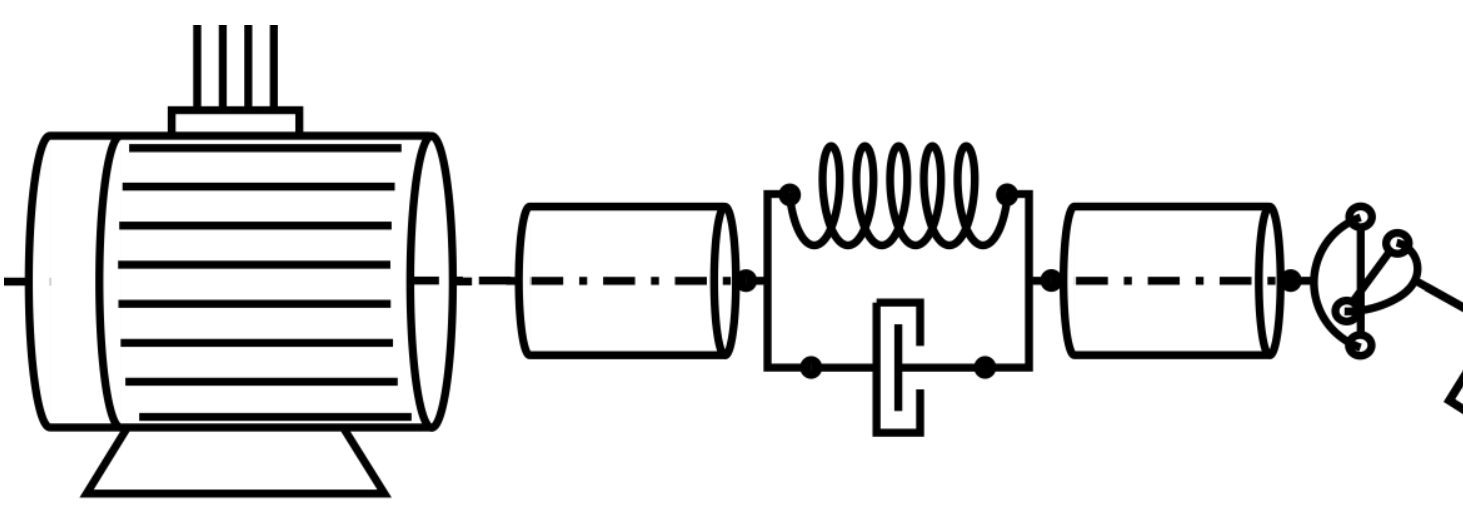

\section{Parameter sampling}

Full factorial $\pm 5 \%$ deviation

$n_{s t}=18$

$n_{d}=20 \quad$ Speed-up: 18

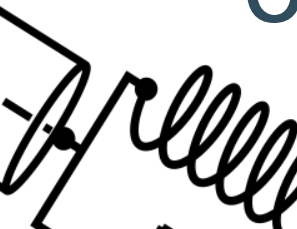
on $R_{r}, L_{m}$ and $k_{2}$

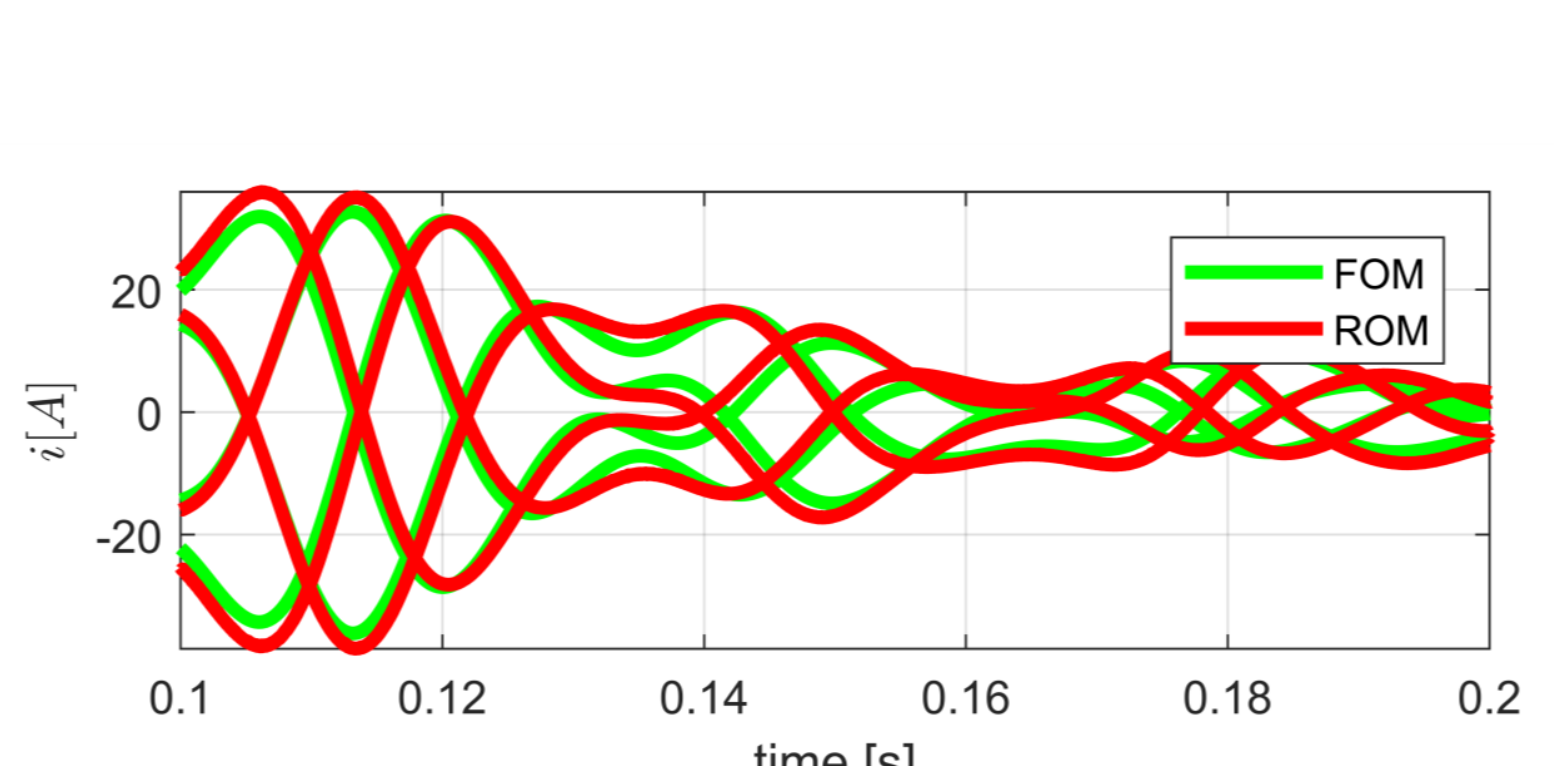

Figure 5 - Numeric demonstration on a mechatronic drivetrain.

\section{HIGHLIGHTS}

- Concurrent state and temporal dimension reduction

- Reduction of a transient simulation (algebraic equations) instead of the model (differential equations)

- Parallel evaluation of certain states at certain time instances

\section{ACKNOWLEDGEMENTS}

The research of W. Rottiers and F. Naets is funded by a grant from the Research Foundation - Flanders (FWO). The Research Fund KU Leuven is also gratefully acknowledged for its support. 